\title{
Measurement of Grüneisen parameter of tissue by photoacoustic spectrometry
}

Da-Kang Yao, Lihong V. Wang

Da-Kang Yao, Lihong V. Wang, "Measurement of Grüneisen parameter of tissue by photoacoustic spectrometry," Proc. SPIE 8581, Photons Plus Ultrasound: Imaging and Sensing 2013, 858138 (4 March 2013); doi: $10.1117 / 12.2004117$

SPIE. Event: SPIE BiOS, 2013, San Francisco, California, United States 


\title{
Measurement of Grüneisen Parameter of Tissue by Photoacoustic Spectrometry
}

\author{
Da-Kang Yao and Lihong V. Wang* \\ Optical Imaging Laboratory, Department of Biomedical Engineering, Washington University in St. \\ Louis, One Brookings Drive, St. Louis, Missouri63130, USA \\ *Corresponding author: LHWANG@WUSTL.EDU
}

\begin{abstract}
The Grüneisen parameter of tissue is a constitutive parameter in photoacoustic tomography. Here, we applied photoacoustic spectrometry (PAS) to directly measure the Grüneisen parameter. In our PAS system, laser pulses at wavelengths between 460 and $1600 \mathrm{~nm}$ were delivered to tissue samples, and photoacoustic signals were detected by a $20 \mathrm{MHz}$ flat water-immersion ultrasonic transducer. By fitting photoacoustic spectra to light absorption spectra, we found that the Grüneisen parameter was 0.73 for porcine subcutaneous fat tissue, and 0.15 for oxygenated bovine red blood cells at room temperature $\left(24^{\circ} \mathrm{C}\right)$.
\end{abstract}

Keywords: photoacoustic tomography, subcutaneous fat, lipid, red blood cell

\section{INTRODUCTION}

The Grüneisen parameter is a constitutive parameter in photoacoustic tomography. In photoacoustic tomography, light pulses are delivered into biological tissue. Once the tissue absorbs the light, it is converted to heat, generating an initial pressure rise due to thermoelastic expansion. The initial pressure gives rise to a photoacoustic signal, which is detected to construct a photoacoustic image. The Grüneisen parameter $\Gamma$ of tissue relates the initial pressure $p_{0}$ to the light absorption by the following expression $[1,2]$,

$$
p_{0}=\Gamma \mu_{a} F,
$$

where $\mu_{a}$ is the absorption coefficient of tissue, and $F$ is the local light fluence. Therefore, the Grüneisen parameter is a critical factor in photoacoustic imaging.

It is necessary to directly measure the Grüneisen parameter of tissue. Scientists can estimate the Grüneisen parameter in terms of the isobaric volume expansion coefficient $\beta$, the specific heat $C_{p}$, and the acoustic speed $v_{s}$ using the following expression: $\Gamma=\beta v_{s}^{2} / C_{p}$, and find that the Grüneisen parameter varies between different types of tissue. However, this estimation usually lacks accuracy. For example, the Grüneisen parameter of fat tissue is estimated to be between 0.7 and 0.9 instead of an exact value [3], causing uncertainty in photoacoustic imaging. In order to minimize the uncertainty, we applied photoacoustic spectrometry (PAS) to directly measure the Grüneisen parameter.

\section{METHODS}

\subsection{Photoacoustic Spectrometry}

We assembled a PAS system to acquire photoacoustic spectra of tissue. Figure 1 is a schematic of the PAS system. An OPO laser system (NT242-SH, Altos Photonics, Bozeman, MT) provides a wavelength tuning range from 460 to 1600 $\mathrm{nm}$. The tunable laser system emits a pulsed laser beam at a repetition rate of $1 \mathrm{kHz}$. The beam diameter is $1 \mathrm{~mm}$ and the pulse width is 5 ns. After passing through a beam sampler (BSF10-B, Thorlabs, Newton, NJ) and a $2.5 \mu \mathrm{m}$ thick

Photons Plus Ultrasound: Imaging and Sensing 2013, edited by Alexander A. Oraevsky, Lihong V. Wang, Proc. of SPIE Vol. 8581, 858138 - C 2013 SPIE · CCC code: 1605-7422/13/\$18 - doi: 10.1117/12.2004117 


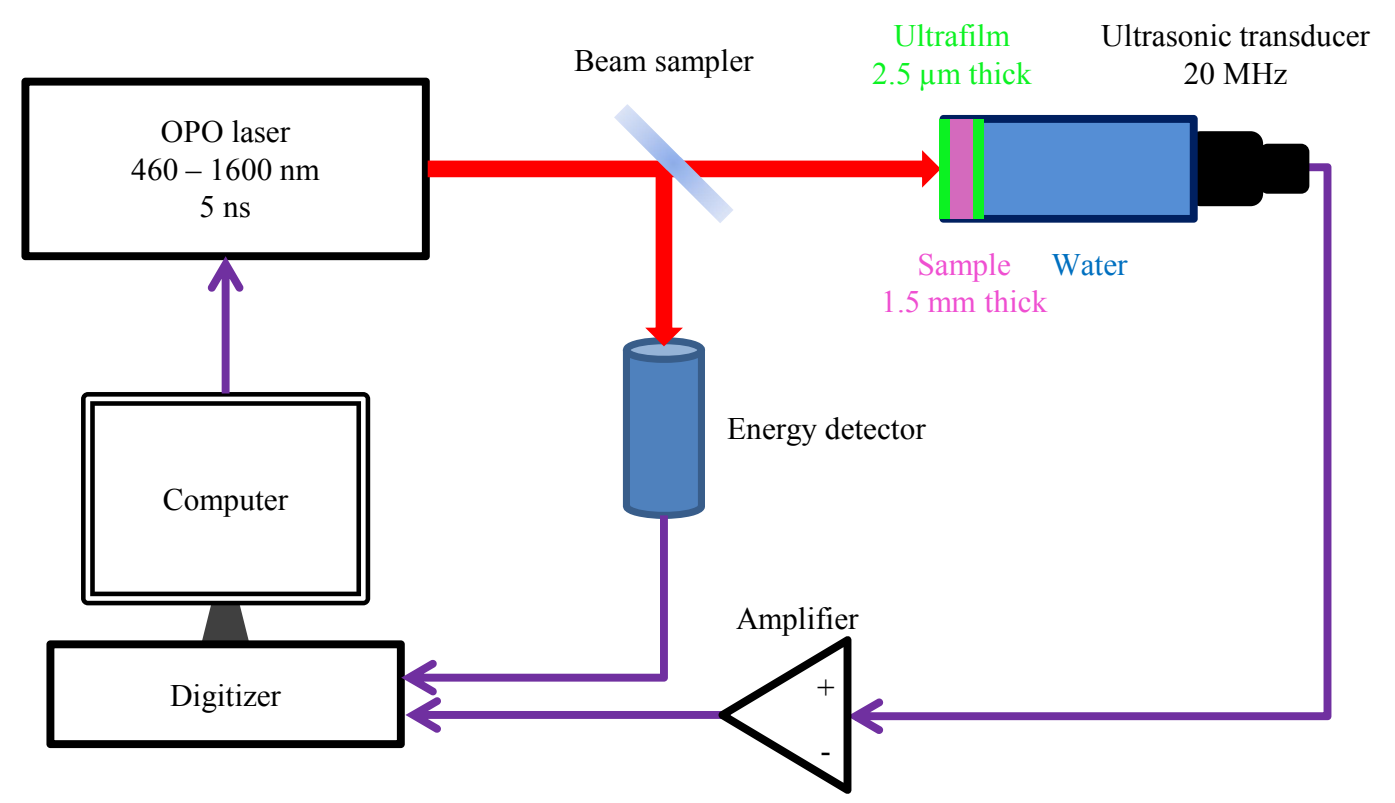

Figure 1.Schematic of the photoacoustic spectrometry system.

ultrafilm (01865-AB, SPI Supplies, West Chester, PA), the laser beam illuminates a $1.5 \mathrm{~mm}$ thick tissue sample in the front part of a cylindrical chamber. A $20 \mathrm{MHz}$ flat ultrasonic transducer (V316-SM,Olympus, Waltham, MA) is installed at the rear of the chamber. Water is filled in the rear part of the chamber for acoustic coupling, and separated from the sample by another piece of ultrafilm. The distance between the sample and the transducer is $33 \mathrm{~mm}$. After detected by the ultrasonic transducer, photoacoustic signals are amplified by an amplifier (ZFL-500LN, Mini-Circuits, Branson, MO). The laser pulse energy is measured by a pair of photodiode detectors (SM05PD2A and SM05PD4A,Thorlabs). Both photoacoustic and photodiode signals are collected by a computer through a 12-bit, $200 \mathrm{MHz}$ digitizer (NI PCI-5124, National Instruments, Austin, TX).

Here, all measurements of photoacoustic spectra were performed at room temperature $\left(24^{\circ} \mathrm{C}\right)$.

\subsection{Photoacoustic Spectrum Fitting}

We obtained the Grüneisen parameter of tissue by fitting the photoacoustic spectra. Photoacoustic spectra are measured in the wavelength range in which the absorption coefficient of tissue is much larger than its reduced scattering coefficient. Thus, the effect of tissue scattering on fluence is neglected. The relation between the peak-to-peak amplitude $(A)$ of a photoacoustic signal, the Grüneisen parameter, the pulse energy $(E)$, and the absorption coefficient of the tissue, is expressed as

$$
A / E=\alpha \Gamma \mu_{a},
$$

where $\alpha$ is a constant in regard to the PAS system, and $A / E$ is a normalized amplitude. The constant $\alpha$ is determined by a system calibration. To reduce errors of photoacoustic spectra, the normalized amplitude is acquired by averaging 10,000 photoacoustic signals at each wavelength. Using Eq. 2, we fit acquired photoacoustic spectra of tissue to its absorption spectra by nonlinear least-squares fitting, in which the Grüneisen parameter is a fitting parameter.

We determined the constant $\alpha$ in Eq. 2 by measuring the photoacoustic spectra of water. A wavelength range from 1200 to $1600 \mathrm{~nm}$ was scanned at a $10 \mathrm{~nm}$ wavelength step size. Knowing the absorption coefficient of water [4] and that the water Grüneisen parameter at $24^{\circ} \mathrm{C}$ is 0.13 , we set $\alpha$ as a fitting parameter. Fitting the photoacoustic spectra of water to its absorption spectrum yielded the setup constant $\alpha$, which was $19.5 \mathrm{mV} \cdot \mathrm{cm} / \mu \mathrm{J}$. 


\section{RESULTS}

\subsection{Grüneisen Parameter of porcine fat tissue}

Porcine subcutaneous fat tissue was cut into $1 \mathrm{~mm}$ thick slices. After a slice adhered to the ultrafilm close to the ultrasonic transducer (Fig. 1), we scanned fat tissue at a wavelength range from 880 to $1000 \mathrm{~nm}$ at a $5 \mathrm{~nm}$ scan step size. A photoacoustic spectrum is shown in Fig. 2. Assuming that light absorption of fat tissue is equal to lipid absorption plus water absorption, we used the Grüneisen parameter and lipid concentration as fitting parameters. A solid curve shown in Fig. 2 is a fit to the absorption spectrum of $98 \%$ lipid and $2 \%$ water $[5,6]$. Using two samples, we found that the Grüneisen parameter of the fat tissue was $0.73 \pm 0.01$ (mean $\pm \mathrm{SD}$ ) at $24^{\circ} \mathrm{C}$.

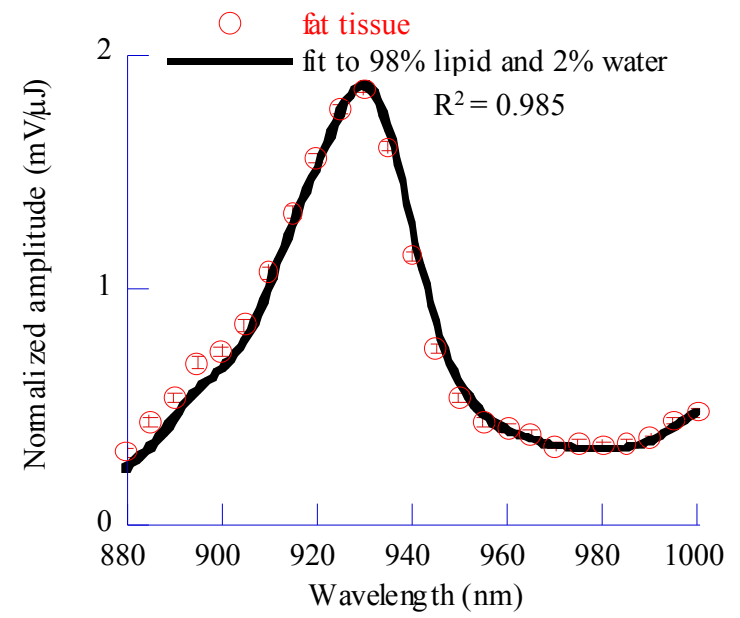

Figure 2. Photoacoustic spectrum of porcine subcutaneous fat tissue. Circles show the normalized amplitude (mean $\pm \mathrm{SE}$ ) of fat tissue versus wavelengths. The solid curve is a fit to the absorption spectrum of $98 \%$ lipid and $2 \%$ water. Here, two fit parameters are the Grüneisen parameter and lipid concentration.

\subsection{Grüneisen Parameter of Bovine Red Blood Cells}

Bovine red blood cells were collected from defibrinated bovine blood (Quad Five, Ryegate, MT) after centrifugation. The cells were washed two times using phosphate buffered saline (PBS) and then suspended in PBS. Hemoglobin concentration of the cell suspension was measured to be $22.6 \mathrm{mg} / \mathrm{ml}$ by a spectrophotometer. To oxygenate the red blood

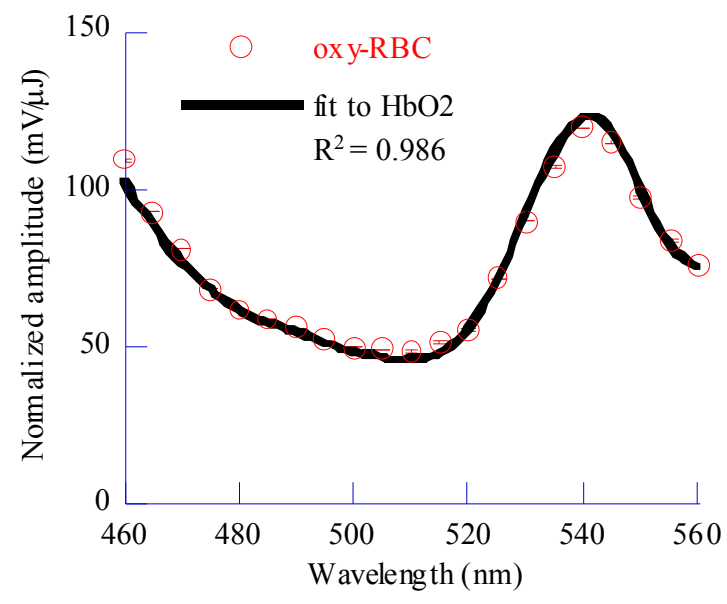

Figure 3. Photoacoustic spectrum of bovine red blood cells (RBC). The cells were suspended in phosphate buffered saline with $22.6 \mathrm{mg} / \mathrm{ml}$ hemoglobin. $\mathrm{RBC}$ was aerated with $\mathrm{O}_{2}$. Circles show the normalized amplitude (mean $\pm \mathrm{SE}$ ) of oxy-RBC versus wavelengths. A solid curve is a fit to the absorption coefficient of oxy-hemoglobin. 
cells, we aerated the cell suspension with oxygen for 1 hour. We scanned the oxygenated cell suspension in a wavelength range from 460 to $560 \mathrm{~nm}$ at a $5 \mathrm{~nm}$ scan step size. A typical photoacoustic spectrum of oxygenated red blood cells is shown in Fig. 3, in which the solid curve is a fit to the absorption spectrum of $22.6 \mathrm{mg} / \mathrm{ml}$ oxygenated hemoglobin [7]. After measuring three oxygenated samples, we found that the Grüneisen parameter was $0.15 \pm 0.01$ (mean $\pm \mathrm{SD}$ ) for oxygenated red blood cells at $24^{\circ} \mathrm{C}$.

\section{DISCUSSION}

Using photoacoustic spectrometry, we developed a method to directly measure the Grüneisen parameter of tissue. Accuracy of the measurement relies on accurate absorption coefficients of the tissue. In our measurements, all sample absorption spectra were collected from published reports. For example, absorption spectra of water and hemoglobin were obtained from a website [7], and absorption spectra of lipid were obtained from both the website and Tsai et al [6]. Since these absorption data are well established, our measurement greatly reduces uncertainty of the Grüneisen parameter. Our method may be generalized to any tissue sample, including tissue of which the absorption coefficient is unknown. If the absorption coefficient of the sample is not known, measuring its absorption coefficient is prerequisite to measuring the Grüneisen parameter by using the method.

Our measurement results support that the Grüneisen parameter varies between different tissues. We found that the Grüneisen parameter was 0.15 for oxygenated red blood cells, and 0.73 for subcutaneous fat tissue at $24{ }^{\circ} \mathrm{C}$, indicating that the Grüneisen parameter of fat tissue is 4.9 times larger than that of oxygenated red blood cells at room temperature. Besides subcutaneous fat tissue, various adipose tissues are located at bone marrow, brain, breast, colon, and liver. Since each adipose tissue has its characteristic compositions, it is very likely that these adipose tissues are different in the Grüneisen parameter. Direct measurement of the Grüneisen parameter of each adipose tissue will allow us to reconstruct accurate photoacoustic images of organs.

\section{CONCLUSIONS}

We directly measured the Grüneisen parameter of tissue by photoacoustic spectrometry to reduce uncertainty in photoacoustic tomography. It is found that the Grüneisen parameter of oxygenated red blood cells is 0.15 at room temperature $\left(24^{\circ} \mathrm{C}\right), 15 \%$ larger than the Grüneisen parameter of water. It is found that the Grüneisen parameter of fat tissue is 0.73 at $24^{\circ} \mathrm{C}, 4.9$ times larger than that of oxygenated red blood cells.

\section{ACKNOWLEDGEMENTS}

This work was sponsored in part by National Institutes of Health grants R01 EB000712, R01 EB008085, R01 CA113453901, U54 CA136398, 5P60 DK02057933, and U54 CA136398. L.W. has a financial interest in Microphotoacoustics, Inc. and Endra, Inc., which, however, did not support this work.

\section{REFERENCES}

[1] Xu, M. and Wang, L. V., "Photoacoustic imaging in biomedicine," Review of Scientific Instruments. 77(4), 041101-22 (2006).

[2] Li, C. and Wang, L. V., "Photoacoustic tomography and sensing in biomedicine," Phys Med Biol. 54(19), R5997 (2009).

[3] Cox, B. T., Laufer, J. G., and Beard, P. C., "The challenges for quantitative photoacoustic imaging," SPIE Proceedings. $7177131-7177139$ (2009).

[4] Kou, L., Labrie, D., and Chylek, P., "Refractive indices of water and ice in the 0.65- to 2.5-microm spectral range," Appl Opt. 32(19), 3531-40 (1993). 
[5] van Veen, R. L. P., et al., "Determination of visible near-IR absorption coefficients of mammalian fat using time- and spatially resolved diffuse reflectance and transmission spectroscopy," Journal of Biomedical Optics. 10(5), 054004-054004 (2005).

[6] Tsai, C.-L., Chen, J.-C., and Wang, W.-J., "Near-infrared Absorption Property of Biological Soft Tissue Constituents," Journal of Medical and Biological Engineering. 21(1), 7-13 (2001).

[7] Prahl, S., "Optical Absorption of Hemoglobin," http://omlc.ogi.edu/spectra/hemoglobin/index.html. 